This is an Accepted Manuscript of an article published by Taylor \& Francis in Stochastic Analysis and Applications on $2^{\text {nd }}$ December 2016, available online:

http://www.tandfonline.com/10.1080/07362994.2016.1248780. 


\title{
A Redundancy Detection Algorithm for Fuzzy Stochastic Multi-Objective Linear Fractional Programming Problems
}

\author{
Rashed Khanjani Shiraz \\ aSchool of Mathematics Science \\ University of Tabriz, Tabriz, Iran \\ E-mail: Rashed.shiraz@gmail.com \\ Vincent Charles ${ }^{b}$ \\ bBuckingham Business School \\ The University of Buckingham \\ Buckingham MK18 1EG, UK \\ v.charles@buckingham.ac.uk

\begin{abstract}
Madjid Tavana, ${ }^{c, d} *$
Distinguished Chair of Business Analytics

E-mail: tavana@lasalle.edu

Web: http://tavana.us/

dBusiness Information Systems Department

Debora Di Caprio e,f

eDepartment of Mathematics and Statistics

York University

Toronto, M3J 1P3, Canada

E-mail: dicaper@mathstat.yorku.ca

fPolo Tecnologico IISS G. Galilei

Via Cadorna 14, 39100, Bolzano, Italy

E-mail: debora.dicaprio@istruzione.it
\end{abstract} \\ 'Business Systems and Analytics Department \\ La Salle University, Philadelphia, PA 19141, USA \\ Faculty of Business Administration and Economics \\ University of Paderborn, D-33098 Paderborn, Germany
}

*Corresponding author at: Business Systems and Analytics Department, Distinguished Chair of Business Analytics, La Salle University, Philadelphia, PA 19141, United States. Tel.: +1 215951 1129; fax: +1 267 2952854. 


\section{ACKNOWLEDGEMENT}

The authors would like to thank the anonymous reviewers and the editor for their insightful comments and suggestions. 


\title{
A Redundancy Detection Algorithm for Fuzzy Stochastic Multi-Objective Linear Fractional Programming Problems
}

\begin{abstract}
The computational complexity of linear and nonlinear programming problems depends on the number of objective functions and constraints involved and solving a large problem often becomes a difficult task. Redundancy detection and elimination provides a suitable tool for reducing this complexity and simplifying a linear or nonlinear programming problem while maintaining the essential properties of the original system. Although a large number of redundancy detection methods have been proposed to simplify linear and nonlinear stochastic programming problems, very little research has been developed for fuzzy stochastic (FS) fractional programming problems. We propose an algorithm that allows to simultaneously detect both redundant objective function(s) and redundant constraint(s) in FS multi-objective linear fractional programming problems. More precisely, our algorithm reduces the number of linear fuzzy fractional objective functions by transforming them in probabilistic-possibilistic constraints characterized by predetermined confidence levels. We present two numerical examples to demonstrate the applicability of the proposed algorithm and exhibit its efficacy.
\end{abstract}

Keywords: Redundancy detection; Fuzzy; Stochastic; Multi-objective; Fractional programming. 


\section{Introduction}

In mathematical programming, a constraint or a variable is redundant if it does not define the solution space, that is, removing the constraint or the variable, the set of feasible solutions remains unaltered. Even though the presence of redundant constraints/variables does not alter the optimal solutions, they usually involve extra computational effort. Thus, redundancy allows to reduce a programming problem to a simpler one from the computational viewpoint without losing any relevant information or property.

Redundancy may occur in the modeling phase of a programming problem and is mostly due to errors in the data input or to the attempt of not omitting any relevant constraints/variables. As a consequence, redundancy is quite common in practical mathematical programming model which makes the development of redundancy detection algorithms a crucial tool in order to obtain efficient solutions to real-life problems. Indeed, redundancy detection algorithms apply to several branches of mathematical programming including linear, integer, nonlinear, and stochastic fractional programming, which have become one of the main planning tools in engineering, business, finance and economics among other disciplines (Bajalinov, 2003; Jeeva et al., 2004; Charles et al., 2010). In particular, fractional programming and stochastic programming have been used to model problems related to investment portfolio, strategic investments, risk management, production planning and scheduling, supply chain optimization, manpower planning, electricity generation capacity, energy planning, environmental and pollution control, telecommunications and so on (Wallace and Ziemba, 2005; Zarif et al., 2013; Ahmed et al., 2014).

We include below a short excursus of the main approaches that have been proposed to identify and remove redundant constraints/variables in mathematical programming, from LP to stochastic fractional programming.

In the mid 1970's, Gal (1975) presented a note on redundancy and linear parametric

programming, followed by Gal and Laberling (1977), who presented an algorithm to identify redundant objective functions in a linear vector maximization problem. Later on, Uthariaraj et al. (1999), Rhymend et al. (1999) and Jacob et al. (2002) proposed algorithms to identify a priori redundant constraints when solving LP problems.

Fractional programming has been studied by many researchers, including Charnes and Cooper (1962), Schaible (1981), Pal and Basu (1995), and Pal et al. (2003), among others. Fractional programming deals with the optimization of one or more ratios of functions subject to 
constraints. Each objective function takes the form of a ratio whose numerator and denominator are both linear. Linear fractional objectives have many applications in financial planning.

In the mid-1950’s, Dantzig (1955) and Charnes and Cooper (1959) first proposed to use stochastic programming to deal with uncertainty in optimization problems. Uncertainty in stochastic programming is usually characterized by the fact that the parameters have known probability distributions. The literature on this topic has been increasingly enriched by the contributions of different disciplines such as operations research and probability theory (Birge, 1995; Wets, 1996; Dupacova, 2002; Birge and Louveaux, 2011). A classification of stochastic programming problems is given by Liu (1999), who introduces the following three groups: (1) the expected value models; (2) Chance-Constrained Programming (CCP) models (Charnes and Cooper, 1959, 1963; Henrion, 2007; Henrion and Strugarek, 2008; Huang, 2007; Yang and Wen, 2005; Pagnoncelli, 2009); and (3) dependent-chance programming models (Liu, 1997; Liu, 2006).

Recently, some more results have been achieved in stochastic fractional programming. Charles and Dutta (2006) proposed an algorithm that identifies redundant objective functions in multi-objective stochastic fractional programming problems, while Charles et al. (2010) provided an algorithm that identifies redundant objective function(s) and redundant constraint(s) simultaneously in multi-objective nonlinear stochastic fractional programming problems.

Despite the large literature existing on linear and nonlinear stochastic fractional programming, not much attention has been paid to fuzzy stochastic (FS) fractional programming problems.

Ever since its introduction by Zadeh (1965), fuzzy set theory has been developing very rapidly and has been applied to a wide variety of real problems. In particular, the possibility theory described by Zadeh (1978) and herein related to fuzzy sets and systems provides a homogeneous framework for the representation of both imprecise and uncertain information. This aspect has been subsequently developed by many researchers; see, among others, Dubois and Prade (1988a, 1988b).

A parallel approach to fuzziness, often considered, is represented by randomness. Indeed, in many complex real-world problems, decision makers (DMs) may encounter a hybrid uncertain environment where fuzziness coexists with randomness. The observed values of data in real-world situations are often inexact, incomplete, vague, ambiguous, or imprecise. Several researchers have combined fuzziness and randomness to model uncertainty. As a consequence, many notions such 
as those of the probability of fuzzy events (Zadeh, 1978), linguistic probabilities (Dubois and Prade, 1980), fuzzy random (FR) variables (Kwakernaak, 1978, 1979; Liu, 2004) and probabilistic sets (Hirota, 1981) have been introduced in the literature. In particular, the concept of a FR variable provides a useful tool to model phenomena where both fuzziness and randomness play a role simultaneously.

In this paper, we consider situations where the parameters are estimated by fuzzy variables whose means are Gaussian random variables and, hence, can be represented by FR variables. We build on the redundancy detection algorithms defined by Charles and Dutta (2006) and Charles et al. (2010) for stochastic fractional programming problems to develop an algorithm that allows to identify and remove redundant objective functions in multi-objective linear FS fractional programming problems. We introduce a probabilistic-possibilistic constrained version of multiobjective fractional programming and rely on the properties of FR variables to operate the necessary conversions of constraints and objective functions to deterministic forms. Hence, the number of linear FS fractional objective functions is reduced by transforming them in probabilistic-possibilistic constraints which are defined by predetermined confidence levels.

The remainder of the paper is organized as follows. In Section 2, we review some basic concepts and definitions related to fuzzy variables and FR variables while, in Section 3, we describe the generic multi-objective fractional programming model and its FS version introducing the probabilistic-possibilistic model. In Section 4, we describe how to obtain the deterministic equivalents of probabilistic-possibilistic constraints and convert objective functions into deterministic constraints. In Section 5, we present the proposed redundancy detection algorithm. In Section 6, we present two numerical examples demonstrating the applicability and the efficacy of the proposed redundancy detection algorithm. Finally, we present our conclusions in Section 7.

\section{Background}

Definition 1: Let $X$ be a universal set and $A \subseteq X$. The fuzzy subset $\tilde{A}$ of $X$ is defined by means of a membership function $\mu_{\tilde{A}}: X \rightarrow[0,1]$ assigning to each element $X \in X$ a real number $\mu_{\tilde{A}}(x) \in[0,1]$ which measures the degree of membership of $x$ in $A$.

Definition 2 (Dubois and Prade, 1980): A fuzzy interval of $L-R$ type is a tuple of the form $\tilde{A}=\left(\alpha, m_{1}, m_{2}, \beta\right)_{L R}$, where $\alpha, \beta, m_{1}, m_{2}$ are non-negative real values, whose membership function is defined as follows: 


$$
\mu_{\tilde{A}}(x)= \begin{cases}L\left(\frac{m_{1}-x}{\alpha}\right), & \text { if } m_{1}-\alpha<x<m_{1} \\ 1, & \text { if } m_{1} \leq x \leq m_{2} \\ R\left(\frac{x-m_{2}}{\beta}\right), & \text { if } m_{2}<x<m_{2}+\beta\end{cases}
$$

The values $\alpha$ and $\beta$ are the left and right spreads, respectively, while $m_{1}$ and $m_{2}$ are the mean values of $\tilde{A}$. The letters $L$ and $R$ stand for non-increasing continuous functions of $[0,1]$ into $[0,1]$ such that $L(0)=R(0)=1$ and $L(1)=R(1)=0$, called the left and right functions, respectively.

Henceforth, we will assume that

$$
L(x)=R(x)= \begin{cases}1-x, & 0 \leq x \leq 1 \\ 0, & \text { otherwise }\end{cases}
$$

If, in particular, $m_{1}=m_{2}=m$, the fuzzy interval of $L-R$ type $\tilde{A}=(\alpha, m, \beta)_{L R}=(\alpha, m, \beta)$ is also called (triangular) fuzzy number.

Definition 3: Let $\tilde{A}$ be a fuzzy interval of $L-R$ type. The support of $\tilde{A}$ is the crisp set $\operatorname{supp}(\tilde{A})=\left\{x \mid \mu_{\tilde{A}}(x) \geq 0\right\}$. The $\gamma$-cut of $\tilde{A}$, denoted by $A_{\gamma}$, is the crisp set $A_{\gamma}=\left\{x \mid \mu_{\tilde{A}}(x) \geq \gamma\right\}$.

As shown in Figure 1, the $\gamma$-cut of a $L-R$ fuzzy interval $\tilde{A}=\left(\alpha, m_{1}, m_{2}, \beta\right)_{L R}$, where $\gamma \in[0,1]$, is a closed interval defined as follows:

$$
A_{\gamma}=\left\{x \mid \mu_{\tilde{A}}(x) \geq \gamma\right\}=\left[A_{\gamma}^{L}, A_{\gamma}^{R}\right]=\left[m_{1}-\alpha L^{-1}(\gamma), m_{2}+\beta R^{-1}(\gamma)\right],
$$

where $A_{\gamma}^{L}$ and $A_{\gamma}^{R}$ are the left and right extreme points, respectively.

\section{Insert Figure 1 Here}

Definition 4 (Fuzzy Arithmetic) (Dubois and Prade, 1980): Let $\tilde{A}=(\alpha, m, \beta)_{L R}$ and $\tilde{B}=(\bar{\alpha}, \bar{m}, \bar{\beta})_{L R}$ be two positive fuzzy numbers. Then:

Addition: $(\alpha, m, \beta)_{L R}+(\bar{\alpha}, \bar{m}, \bar{\beta})_{L R}=(\alpha+\bar{\alpha}, m+\bar{m}, \beta+\bar{\beta})_{L R}$

Subtraction: $(\alpha, m, \beta)_{L R}-(\bar{\alpha}, \bar{m}, \bar{\beta})_{L R}=(\alpha+\bar{\beta}, m-\bar{m}, \beta+\bar{\alpha})_{L R}$

Definition 5 (Extension Principle) (Zimmermann, 1996): Assume that $X$ is the Cartesian product 
of $r$ universes ( $r$ is a real number), i.e. $X=X_{1} \times \ldots \times X_{r}$, and that $\tilde{A}_{1}, \ldots, \tilde{A}_{r}$ are $r$ fuzzy subsets of $X_{1}, \ldots, X_{r}$, respectively. Let $f$ be a mapping from $X$ to a universe $Y$, i.e. $y=f\left(x_{1}, \ldots, x_{r}\right)$. The extension principle enables us to define a fuzzy subset $\tilde{B}$ of $Y$ by using the following membership function:

$$
\mu_{\tilde{B}}(y)= \begin{cases}\sup _{\left(x_{1}, \ldots, x_{r}\right) \in f^{-1}(y)} \min \left\{\mu_{\tilde{A}_{1}}\left(x_{1}\right), \ldots, \mu_{\tilde{A}_{r}}\left(x_{r}\right)\right\}, & f^{-1}(y) \neq \varnothing \\ 0, & \text { otherwise }\end{cases}
$$

where $f^{-1}$ denotes the inverse of $f$.

Dubois and Prade (1980) modified the extension principle and used the algebraic sum and product instead of sup and min, respectively.

Definition 6: A fuzzy variable $\xi$ is a mapping from an abstract space onto the real line.

Definition 7 (Zadeh, 1978; Zimmermann, 1996): Let $(\Theta, P(\Theta)$, Pos ) be a possibility space where $\Theta$ is a non-empty set involving all possible events, and $P(\Theta)$ is the power set of $\Theta$. For every $A \in P(\Theta)$, there is a non-negative number $\operatorname{Pos}(A)$, so-called a possibility measure, satisfying the following axioms:

(i) $\quad \operatorname{Pos}(\varnothing)=0, \quad \operatorname{Pos}(\Theta)=1$;

(ii) for every $A, B \in P(\Theta), A \subseteq B$ implies $\operatorname{Pos}(A) \leq \operatorname{Pos}(B)$;

(iii) for every subset $\left\{A_{w}: w \in W\right\} \subseteq P(\Theta), \operatorname{Pos}\left(\bigcup_{w} A_{w}\right)=\operatorname{Sup}_{w} \operatorname{Pos}\left(A_{w}\right)$.

The elements of $P(\Theta)$ are also called fuzzy events.

Definition 8: Let $\xi$ be a fuzzy variable on the possibility space $(\Theta, P(\Theta), \operatorname{Pos}), \mu_{\xi}: \Re \rightarrow[0,1]$ be the membership function of $\xi$ and $r$ be a real number. The possibility of the fuzzy event $\{\xi \geq r\}$ is given by $\operatorname{Pos}\{\xi \geq r\}=\operatorname{Sup}_{t \geq r} \mu_{\xi}(t)$.

Definition 9 (Liu and Liu, 2003): A FR variable is a random variable $\xi$ on a probability space $(\Omega, \mathcal{A}, \operatorname{Pr})$ whose values are fuzzy numbers.

Each value $\xi(\omega)$ (with $\omega \in \Omega$ ) taken by a FR variable has a membership $\mu_{\xi(\omega)}$. In particular, a FR variable of $L-R$ type takes values in the set of all fuzzy numbers of $L-R$ type. 
In the following, we will consider only this kind of FR variables. The associated membership functions are described below.

$$
\mu_{\xi(\omega)}(x)= \begin{cases}L\left(\frac{m(\omega)-x}{\alpha}\right), & x \leq m(\omega), \\ 1 & x=m(\omega), \\ R\left(\frac{x-m(\omega)}{\beta}\right), & x \geq m(\omega) .\end{cases}
$$

where $m(\omega)$ is the normally distributed random variable.

Figure 2 shows a triangular FR variable, that is, a triangular fuzzy variable whose mean value is a normally-distributed random variable $\left(\bar{m} \sim N\left(\mu, \sigma^{2}\right)\right)$.

\section{Insert Figure 2 Here}

Proposition 1 (Liu, 2004): Let $\xi$ be a FR vector and, for every $j=1,2, \ldots, n$, let $\boldsymbol{g}_{j}$ be a realvalued continuous function. Then, the possibility $\operatorname{Pos}\left\{g_{j}(\xi(\omega)) \leq 0, j=1, \ldots, n\right\}$ is a random variable.

Proposition 2 (Sakawa, 1993): Let $\tilde{A}_{1}$ and $\widetilde{A}_{2}$ be two independent fuzzy numbers with continuous membership functions. For a given confidence level $\gamma \in[0,1]$, we have:

$$
\operatorname{Pos}\left\{\tilde{A}_{1} \geq \tilde{A}_{2}\right\} \geq \gamma \quad \Leftrightarrow \quad A_{1, \gamma}^{R} \geq A_{2, \gamma}^{L}
$$

where, for $l=1,2, A_{l, \gamma}^{L}$ and $A_{l, \gamma}^{R}$ are the left and right extreme points of the $\gamma$-cut $\left[A_{l, \gamma}^{L}, A_{l, \gamma}^{R}\right]$, respectively. $\operatorname{Pos}\left\{\widetilde{A}_{1} \geq \widetilde{A}_{2}\right\}$ represents the degree of possibility that $\tilde{A}_{1} \geq \tilde{A}_{2}$.

\section{Multi-objective fuzzy stochastic fractional programming}

In this section we develop an imprecise fractional programming formulation to deal with the randomness of fuzzy parameters in a possibility space $(\Theta, P(\Theta)$,Pos $)$. First, we define a generic multi-objective fractional programming model. Hence, we move to a multi-objective FS fractional programming setting introducing the Probability-Possibility Constrained Programming (PPCP) model. Note that, in the following, we will also use prob-pos to shorten Probability-Possibility.

The generic multi-objective fractional programming model is defined as follows: 
$\operatorname{Max}\left[\frac{\sum_{j=1}^{n} \tilde{\bar{c}}_{1 j} x_{j}+\tilde{\bar{\alpha}}_{1}}{\sum_{j=1}^{n} \tilde{\bar{d}}_{1 j} x_{j}+\tilde{\bar{\beta}}}, \frac{\sum_{j=1}^{n} \tilde{\overline{\bar{\beta}}}_{2 j} x_{j}+\tilde{\bar{\alpha}}_{2}}{\sum_{j=1}^{n} \tilde{\bar{d}}_{2 j} x_{j}+\tilde{\bar{\beta}}_{2}}, \ldots, \frac{\sum_{j=1}^{n} \tilde{\bar{c}}_{m j} x_{j}+\tilde{\bar{\alpha}}_{m}}{\sum_{j=1}^{n} \tilde{\bar{d}}_{m j} x_{j}+\tilde{\bar{\beta}}_{m}}\right]$

S.t.

$\sum_{j=1}^{n} \tilde{\bar{a}}_{r j}^{(1)} x_{j} \leq \tilde{\bar{b}}_{r}^{(1)}, \quad r=1,2, \ldots, s$,

$\sum_{j=1}^{n} a_{r j}^{(2)} x_{j} \leq b_{r}^{(2)}, \quad r=1,2, \ldots, s$,

$x_{j} \geq 0, \quad j=1,2, \ldots, n$.

where

- $\quad \tilde{\bar{c}}_{i j}, \tilde{\bar{d}}_{i j}, \tilde{\bar{\alpha}}_{i}, \tilde{\bar{\beta}}_{i} \tilde{\bar{a}}_{r j}^{(1)}$ and $\tilde{\bar{b}}_{r}^{(1)}$ are FR variables for every $i=1, \ldots, m$, every $j=1, \ldots, n$ and every $r=1, \ldots, s$

- $a_{r j}^{(2)}$ and $b_{r}^{(2)}$ are deterministic parameters for every $j=1, \ldots, n$ and every $r=1, \ldots, S$.

Model (2) can be rewritten as a multi-objective FS fractional programming problem, which we will refer to as PPCP model, as follows:

$\operatorname{Max}\left[\lambda_{1}, \lambda_{2}, \ldots, \lambda_{m}\right]$

s.t.

$\left\{\begin{array}{l}\operatorname{Pr}\left[\operatorname{Pos}\left[\frac{\sum_{j=1}^{n} \tilde{\bar{c}}_{i j} x_{j}+\tilde{\bar{\alpha}}_{i}}{\sum_{j=1}^{n} \tilde{\bar{d}}_{i j} x_{j}+\tilde{\bar{\beta}}_{i}} \geq \lambda_{i}\right] \geq \delta_{i}\right] \geq \gamma_{i}, i=1,2, \ldots, m, \\ \operatorname{Pr}\left[\operatorname{Pos}\left[\sum_{j=1}^{n} \tilde{\bar{a}}_{r j}^{(1)} x_{j} \leq \tilde{\bar{b}}_{r}^{(1)}\right] \geq u_{r}\right] \geq p_{r}, \quad r=1,2, \ldots, s, \\ \sum_{j=1}^{n} a_{r j}^{(2)} x_{j} \leq b_{r}^{(2)}, \quad r=1,2, \ldots, s, \\ x_{j} \geq 0, \quad j=1,2, \ldots, n .\end{array}\right.$

where

- $\quad \tilde{\overline{\bar{c}_{i j}}}, \tilde{\overline{\bar{d}_{i j}}}, \tilde{\bar{\alpha}}_{i}, \tilde{\bar{\beta}}_{i}, \tilde{\bar{a}}_{r j}^{(1)}$ and $\tilde{\bar{b}}_{r}^{(1)}$ are FR variables for every $i=1, \ldots, m$, every $j=1, \ldots, n$ and every 


$$
r=1, \ldots, s
$$

- $\quad a_{r j}^{(2)}$ and $b_{r}^{(2)}$ are deterministic parameters for every $j=1, \ldots, n$ and every $r=1, \ldots, S$;

- $\delta_{i}$ and $\gamma_{i}$, with $i=1, \ldots, m$, and $u_{r}$ and $p_{r}$, with $r=1,2, \ldots, s$, are the predetermined confidence levels.

Note that:

$$
S=\left\{\mathbf{x}=\left(x_{1}, \ldots, x_{n}\right) \mid \forall r=1, \ldots, s, \sum_{j=1}^{n} \tilde{\bar{a}}_{r j}^{(1)} x_{j} \leq \tilde{\bar{b}}_{r}^{(1)} \text { and } \sum_{j=1}^{n} a_{r j}^{(2)} x_{j} \leq b_{r}^{(2)}, \forall j=1, \ldots, n, x_{j} \geq 0\right\}
$$

is a non-empty, convex and compact subset of $\mathfrak{R}^{n}$.

Recall that Pos[.] denotes the possibility of the event described in [·], while $\operatorname{Pr}[\cdot]$ denotes the probability of the event in [·].

\section{Deterministic equivalents and conversions}

\subsection{Deterministic equivalents of probabilistic-possibilistic constraints}

One way to solve the prob-pos constrained multi-objective FS fractional programming problem described by model (3), i.e., the PPCP model, is to convert its constraints into their respective crisp equivalents.

For every $j=1, \ldots, n$ and every $r=1, \ldots, s$, the variable $\tilde{a}_{r j}^{(1)}$ appearing in the second set of constraints of PPCP model (3) is assumed to be a FR variable. Thus, $\tilde{a}_{r j}^{(1)}$ can be written as $\tilde{\bar{a}}_{r j}^{(1)}=\left(\alpha_{r j}, \bar{a}_{r j}^{(1)}, \beta_{r j}\right)_{L R}$, where, for every $j=1, \ldots, n$ and every $r=1, \ldots, S, \bar{a}_{r j}^{(1)}, \alpha_{r j}$ and $\beta_{r j}$ stand for the center value, the left tail and the right tail, respectively. In particular, $\bar{a}_{r j}^{(1)} \sim N\left(a_{r j}^{(1)}, \sigma_{r j}^{2}\right)$, where $a_{r j}^{(1)}$ denotes the mean and $\sigma_{r j}^{2}$ the variance.

Theorem 1: Let $\widetilde{a}_{j}=\left(a_{j}^{\alpha}, a_{j}^{m}, a_{j}^{\beta}\right)_{L R}$, with $j=1, \ldots, n$, and $\widetilde{\bar{b}}=\left(b^{\alpha}, b^{m}, b^{\beta}\right)_{L R}$ be FR numbers such that $a_{j}^{m} \sim N\left(a_{j}, \sigma_{j}^{2}\right)$ and $b^{m} \sim N\left(b, \hat{\sigma}^{2}\right)$ have a normal distribution. Let $u$ and $p$ be two predetermined confidence levels. The following are equivalent:

(a) $\operatorname{Pr}\left[\operatorname{Pos}\left[\sum_{j=1}^{n} \tilde{\bar{a}}_{j} x_{j} \leq \tilde{\bar{b}}\right] \geq u\right] \geq p$

(b) $\sum_{j=1}^{n}\left(a_{j}-L^{-1}(u) a_{j}^{\alpha}\right) x_{j}+\Phi^{-1}(p) \sqrt{\sum_{j=1}^{n} \sigma_{j}^{2} x_{j}^{2}+\hat{\sigma}^{2}} \leq b+R^{-1}(u) b^{\beta}$ 
Proof: By Definition 2, we can assume the FR numbers $\tilde{\bar{a}}_{j}$, with $j=1, \ldots, n$, and $\tilde{\bar{b}}$ to be characterized, respectively, by the following membership functions:

For every $j=1, \ldots, n$,

$\mu_{\widetilde{a}_{j}}(t)=\left\{\begin{array}{l}L\left(\frac{a_{j}^{m}-t}{a_{j}^{\alpha}}\right), \text { if } a_{j}^{m}-a_{j}^{\alpha}<t \leq a_{j}^{m} \\ R\left(\frac{t-a_{j}^{m}}{a_{j}^{\beta}}\right), \text { if } a_{j}^{m} \leq t<a_{j}^{m}+a_{j}^{\beta}\end{array}\right.$

and

$$
\mu_{\tilde{b}}(t)=\left\{\begin{array}{l}
L\left(\frac{b^{m}-t}{b^{\alpha}}\right), \text { if } b^{m}-b^{\alpha}<t \leq b^{m} \\
R\left(\frac{t-b^{m}}{b^{\beta}}\right), \text { if } b^{m} \leq t<b^{m}+b^{\beta}
\end{array}\right.
$$

Using the extension principle (see Definition 5), the fuzzy number $\sum_{j=1}^{n} \tilde{\bar{a}}_{j} x_{j}$, where $x_{j} \geq 0$ for every $j=1, \ldots, n$, can be associated with the following membership function:

$$
\mu_{\sum_{j=1}^{n} \tilde{a}_{j} x_{j}}(t)=\left\{\begin{array}{l}
\left(\frac{\sum_{j=1}^{n} a_{j}^{m} x_{j}-t}{\sum_{j=1}^{n} a_{j}^{\alpha} x_{j}}\right), \text { if } \sum_{j=1}^{n} a_{j}^{m} x_{j}-\sum_{j=1}^{n} a_{j}^{\alpha} x_{j}<t \leq \sum_{j=1}^{n} a_{j}^{m} x_{j} \\
R\left(\frac{t-\sum_{j=1}^{n} a_{j}^{m} x_{j}}{\sum_{j=1}^{n} a_{j}^{\beta} x_{j}}\right), \text { if } \sum_{j=1}^{n} a_{j}^{m} x_{j} \leq t<\sum_{j=1}^{n} a_{j}^{m} x_{j}+\sum_{j=1}^{n} a_{j}^{\beta} x_{j}
\end{array}\right.
$$

Therefore, $\sum_{j=1}^{n} \tilde{\bar{a}}_{j} x_{j}$ corresponds to the triple $\left(\sum_{j=1}^{n} a_{j}^{\alpha} x_{j}, \sum_{j=1}^{n} a_{j}^{m} x_{j}, \sum_{j=1}^{n} a_{j}^{\beta} x_{j}\right)_{L R}$, while the following intervals describe the $u$-cut (see Definition 3) of $\sum_{j=1}^{n} \tilde{\bar{a}}_{j} x_{j}$ and $\widetilde{\widetilde{b}}$, respectively:

$$
\left[\left(\sum_{j=1}^{n} \tilde{\bar{a}}_{j} x_{j}\right)_{u}^{L},\left(\sum_{j=1}^{n} \widetilde{\bar{a}}_{j} x_{j}\right)_{u}^{R}\right]=\left[\sum_{j=1}^{n} a_{j}^{m} x_{j}-L^{-1}(u) \sum_{j=1}^{n} a_{j}^{\alpha} x_{j}, \sum_{j=1}^{n} a_{j}^{m} x_{j}+R^{-1}(u) \sum_{j=1}^{n} a_{j}^{\beta} x_{j}\right]
$$


$\left[(\tilde{\bar{b}})_{u}^{L},(\tilde{\bar{b}})_{u}^{R}\right]=\left[b^{m}-L^{-1}(u) b^{\alpha}, b^{m}+R^{-1}(u) b^{\beta}\right]$.

By Proposition 2, the constraint $\operatorname{Pr}\left[\operatorname{Pos}\left[\sum_{j=1}^{n} \tilde{\bar{a}}_{j} x_{j} \leq \widetilde{\bar{b}}\right] \geq u\right] \geq p$ is equivalent to the following:

$\operatorname{Pr}\left[\left(\sum_{j=1}^{n} \tilde{\bar{a}}_{j} x_{j}\right)_{u}^{L} \leq(\widetilde{\bar{b}})_{u}^{R}\right] \geq p$,

which, in turn, is equivalent to:

$$
\begin{aligned}
\operatorname{Pr}\left[b^{m}+R^{-1}(u) b^{\beta}\right. & \left.\geq \sum_{j=1}^{n}\left(a_{j}^{m}-L^{-1}(u) a_{j}^{\alpha}\right) x_{j}\right] \geq p . \\
\text { Letting } \tilde{h} & =\sum_{j=1}^{n}\left(a_{j}^{m}-L^{-1}(u) a_{j}^{\alpha}\right) x_{j}-b^{m}-R^{-1}(u) b^{\beta} \text {, we have }
\end{aligned}
$$

$\operatorname{Pr}[\tilde{h} \leq 0] \geq p$

Obviously, $\tilde{h}$ also has a normal distribution with:

$$
\mu_{\tilde{h}}=E(\tilde{h})=\sum_{j=1}^{n}\left(a_{j}-L^{-1}(u) a_{j}^{\alpha}\right) x_{j}-b-R^{-1}(u) b^{\beta} \quad \text { and } \quad \sigma_{\tilde{h}}^{2}=\sum_{j=1}^{n} \sigma_{j}^{2} x_{j}^{2}+\hat{\sigma}^{2} .
$$

Finally, we claim that:

$$
\operatorname{Pr}[\tilde{h} \leq 0] \geq p \Leftrightarrow E[\tilde{h}]+\Phi^{-1}(p) \sigma_{\tilde{h}} \leq 0
$$

where $\Phi^{-1}$ is the inverse of the CDF.

In fact:

$$
\operatorname{Pr}[\tilde{h} \geq 0]=\operatorname{Pr}\left[\frac{\tilde{h}-E[\tilde{h}]}{\sigma_{\tilde{h}}} \geq \frac{-E[\tilde{h}]}{\sigma_{\tilde{h}}}\right]=\operatorname{Pr}\left[\frac{E[\tilde{h}]-\tilde{h}}{\sigma_{\tilde{h}}} \leq \frac{E[\tilde{h}]}{\sigma_{\tilde{h}}}\right]=\Phi\left(\frac{E[\tilde{h}]}{\sigma_{\tilde{h}}}\right)
$$

From which it follows that:

$$
\begin{aligned}
& \operatorname{Pr}[\tilde{h} \leq 0] \geq p \Leftrightarrow 1-\operatorname{Pr}[\tilde{h} \geq 0] \geq p \Leftrightarrow \operatorname{Pr}[\tilde{h} \geq 0] \leq 1-p \Leftrightarrow \Phi\left(\frac{E[\tilde{h}]}{\sigma_{\tilde{h}}}\right) \leq 1-p \Leftrightarrow \\
& \frac{E[\tilde{h}]}{\sigma_{\tilde{h}}} \leq \Phi^{-1}(1-p) \Leftrightarrow E[\tilde{h}] \leq \Phi^{-1}(1-p) \sigma_{\tilde{h}} \Leftrightarrow E[\tilde{h}]-\Phi^{-1}(1-p) \sigma_{\tilde{h}} \leq 0 \Leftrightarrow E[\tilde{h}]+\Phi^{-1}(p) \sigma_{\tilde{h}} \leq 0
\end{aligned}
$$


Therefore, $\operatorname{Pr}[\tilde{h} \leq 0] \geq p$ is equivalent to

$$
\sum_{j=1}^{n}\left(a_{j}-L^{-1}(u) a_{j}^{\alpha}\right) x_{j}+\Phi^{-1}(p) \sqrt{\sum_{j=1}^{n} \sigma_{j}^{2} x_{j}^{2}+\hat{\sigma}^{2}} \leq b+R^{-1}(u) b^{\beta} .
$$

\subsection{Conversion of objective functions into deterministic constraints}

In this section, we use Proposition 2 and Theorem 1 to obtain a deterministic programming version of model (3). The main feature of the model is that it takes into account the probability distribution of the objective functions by maximizing the lower allowable limit of the objective functions subject to chance constraints where both the numerator and denominator coefficients are FR variables. As in Subsection 4.1, we will work with the PPCP model (3), that is:

$\operatorname{Max}\left[\lambda_{1}, \lambda_{2}, \ldots, \lambda_{m}\right]$

s.t.

$$
\left\{\begin{array}{l}
\operatorname{Pr}\left[\operatorname{Pos}\left[\frac{\sum_{j=1}^{n} \tilde{\bar{c}}_{i j} x_{j}+\tilde{\bar{\alpha}}_{i}}{\sum_{j=1}^{n} \tilde{\bar{d}}_{i j} x_{j}+\widetilde{\bar{\beta}}_{i}} \geq \lambda_{i}\right] \geq \delta_{i}\right] \geq \gamma_{i}, \quad i=1,2, \ldots, m, \\
\operatorname{Pr}\left[\operatorname{Pos}\left[\sum_{j=1}^{n} \tilde{\bar{a}}_{r j}^{(1)} x_{j} \leq \tilde{\bar{b}}_{r j}^{(1)}\right] \geq u_{r}\right] \geq p_{r}, \quad r=1,2, \ldots, s, \\
\sum_{j=1}^{n} a_{r j}^{(2)} x_{j} \leq b_{r}^{(2)}, \quad r=1,2, \ldots, s, \\
x_{j} \geq 0, \quad j=1,2, \ldots, n,
\end{array}\right.
$$

where

- $\quad \tilde{\overline{\bar{c}_{i j}}}, \tilde{\tilde{\bar{d}}_{i j}}, \tilde{\bar{\alpha}}_{i}, \tilde{\bar{\beta}}_{i}, \tilde{\bar{a}}_{r j}^{(1)}$ and $\tilde{\bar{b}}_{r}^{(1)}$ are FR variables for every $i=1, \ldots, m$, every $j=1, \ldots, n$ and every

$$
r=1, \ldots, s \text {; }
$$

- $a_{r j}^{(2)}$ and $b_{r}^{(2)}$ are deterministic parameters for every $j=1, \ldots, n$ and every $r=1, \ldots, S$.

More precisely, for every $i=1, \ldots, m$ and every $j=1, \ldots, n$, let

$$
\tilde{\bar{c}}_{i j}=\left(c_{i j}^{\alpha}, c_{i j}^{m}, c_{i j}^{\beta}\right)_{L R}, \tilde{\bar{d}}_{i j}=\left(d_{i j}^{\alpha}, d_{i j}^{m}, d_{i j}^{\beta}\right)_{L R}, \tilde{\bar{\alpha}}_{i}=\left(\alpha_{i}^{\alpha}, \alpha_{i}^{m}, \alpha_{i}^{\beta}\right)_{L R}, \tilde{\bar{\beta}}_{i}=\left(\beta_{i}^{\alpha}, \beta_{i}^{m}, \beta_{i}^{\beta}\right)_{L R} \text {, }
$$


where $c_{i j}^{m}, \quad d_{i j}^{m}, \quad \alpha_{i}^{m}$ and $\beta_{i}^{m}$ are normally distributed, that is, $c_{i j}^{m}: N\left(c_{i j}, \sigma_{c i j}^{2}\right)$, $d_{i j}^{m}: N\left(d_{i j}, \sigma_{d i j}^{2}\right), \alpha_{i}^{m}: N\left(\alpha_{i}, \sigma_{\alpha i}^{2}\right)$ and $\beta_{i}^{m}: N\left(\beta_{i}, \sigma_{\beta i}^{2}\right)$. Hence, $c_{i j}, \alpha_{i}\left(\right.$ resp. $\left.d_{i j}, \beta_{i}\right)$ and $\sigma_{c i j}^{2}, \sigma_{\alpha i}^{2}$ (resp. $\sigma_{d i j}^{2}, \sigma_{\beta i}^{2}$ ) are the mean and the variance of $c_{i j}^{m}, \alpha_{i}^{m}$ (resp. $d_{i j}^{m}, \beta_{i}^{m}$ ), respectively.

For every $i=1, \ldots, m$, the unknown parameter $\lambda_{i}$, which is less than or equal to $\frac{\sum_{j=1}^{n} \tilde{\bar{c}}_{i j} x_{j}+\tilde{\bar{\alpha}}_{i}}{\sum_{j=1}^{n} \tilde{\bar{d}}_{i j} x_{j}+\tilde{\bar{\beta}}_{i}}$, satisfies the following inequality:

$$
\sum_{j=1}^{n} \widetilde{\bar{c}}_{i j} x_{j}+\widetilde{\bar{\alpha}}_{i}-\lambda_{i}\left(\sum_{j=1}^{n} \widetilde{\bar{d}}_{i j} x_{j}+\tilde{\bar{\beta}}_{i}\right) \geq 0 \text {. }
$$

By Proposition 2, the $i$ th inequality of the first set of constraints in model (7) is equivalent to the following inequality:

$$
\begin{aligned}
\operatorname{Pos}\left[\sum_{j=1}^{n} \tilde{\bar{c}}_{i j} x_{j}+\tilde{\bar{\alpha}}_{i}-\lambda_{i}\left(\sum_{j=1}^{n} \tilde{\bar{d}}_{i j} x_{j}+\tilde{\bar{\beta}}_{i}\right) \geq 0\right] \geq \delta_{i} \Leftrightarrow \\
\quad \lambda_{i}\left[\sum_{j=1}^{n}\left(d_{i j}^{m}-L^{-1}\left(\delta_{i}\right) d_{i j}^{\alpha}\right) x_{j}+\beta_{i}^{m}-L^{-1}\left(\delta_{i}\right) \beta_{i}^{\alpha}\right]-\sum_{j=1}^{n}\left(c_{i j}^{m}+R^{-1}\left(\delta_{i}\right) c_{i j}^{\beta}\right) x_{j} \leq \alpha_{i}^{m}+R^{-1}\left(\delta_{i}\right) \alpha_{i}^{\beta}
\end{aligned}
$$

For every $i=1, \ldots, m$, let

$$
\tilde{f_{i}}=\lambda_{i}\left[\sum_{j=1}^{n}\left(d_{i j}^{m}-L^{-1}\left(\delta_{i}\right) d_{i j}^{\alpha}\right) x_{j}+\beta_{i}^{m}-L^{-1}\left(\delta_{i}\right) \beta_{i}^{\alpha}\right]-\sum_{j=1}^{n}\left(c_{i j}^{m}+R^{-1}\left(\delta_{i}\right) c_{i j}^{\beta}\right) x_{j}-\alpha_{i}^{m}-R^{-1}\left(\delta_{i}\right) \alpha_{i}^{\beta}
$$

The expected value and variance of $\tilde{f_{i}}$ are obtained as follows:

$$
\begin{aligned}
& E\left[\tilde{f}_{i}\right]=E\left[\lambda_{i}\left[\sum_{j=1}^{n}\left(d_{i j}^{m}-L^{-1}\left(\delta_{i}\right) d_{i j}^{\alpha}\right) x_{j}+\beta_{i}^{m}-L^{-1}\left(\delta_{i}\right) \beta_{i}^{\alpha}\right]-\sum_{j=1}^{n}\left(c_{i j}^{m}+R^{-1}\left(\delta_{i}\right) c_{i j}^{\beta}\right) x_{j}-\alpha_{i}^{m}-R^{-1}\left(\delta_{i}\right) \alpha_{i}^{\beta}\right] \\
& =\lambda_{i}\left[\sum_{j=1}^{n}\left(d_{i j}-L^{-1}\left(\delta_{i}\right) d_{i j}^{\alpha}\right) x_{j}+\beta_{i}-L^{-1}\left(\delta_{i}\right) \beta_{i}^{\alpha}\right]-\sum_{j=1}^{n}\left(c_{i j}+R^{-1}\left(\delta_{i}\right) c_{i j}^{\beta}\right) x_{j}-\alpha_{i}-R^{-1}\left(\delta_{i}\right) \alpha_{i}^{\beta} \\
& \operatorname{Var}\left[\tilde{f}_{i}\right]= \\
& \operatorname{Var}\left[\lambda_{i}\left[\sum_{j=1}^{n}\left(d_{i j}^{m}-L^{-1}\left(\delta_{i}\right) d_{i j}^{\alpha}\right) x_{j}+\beta_{i}^{m}-L^{-1}\left(\delta_{i}\right) \beta_{i}^{\alpha}\right]-\left(\sum_{j=1}^{n}\left(c_{i j}^{m}+R^{-1}\left(\delta_{i}\right) c_{i j}^{\beta}\right) x_{j}+\alpha_{i}^{m}+R^{-1}\left(\delta_{i}\right) \alpha_{i}^{\beta}\right)\right] \\
& =\lambda_{i}^{2} \sum_{j=1}^{n} \sigma_{d i j}^{2} x_{j}^{2}+\lambda_{i}^{2} \sigma_{\beta i}^{2}+\sum_{j=1}^{n} \sigma_{c i j}^{2} x_{j}^{2}+\sigma_{\alpha i}^{2} .
\end{aligned}
$$


Reasoning as in the proof of Theorem 1 (see Claim at the end of the proof), we have:

$$
\operatorname{Pr}\left[\tilde{f}_{i} \leq 0\right] \geq \gamma_{i} \Leftrightarrow E\left[\tilde{f}_{i}\right]+\Phi^{-1}\left(\gamma_{i}\right) \sigma_{\tilde{f}_{i}} \leq 0
$$

Thus, the ith prob-pos constraint is converted into the following:

$$
\begin{aligned}
& \operatorname{Pr}\left[\operatorname{Pos}\left[\sum_{j=1}^{n} \tilde{\bar{c}}_{i j} x_{j}+\tilde{\bar{\alpha}}_{i}-\lambda_{i}\left[\sum_{j=1}^{n} \tilde{\bar{d}}_{i j} x_{j}+\tilde{\bar{\beta}}_{i}\right] \geq 0\right] \geq \delta_{i}\right] \geq \gamma_{i} \Leftrightarrow \operatorname{Pr}\left[\tilde{f}_{i} \leq 0\right] \geq \gamma_{i} \Leftrightarrow \\
& \lambda_{i}\left[\sum_{j=1}^{n}\left(d_{i j}-L^{-1}\left(\delta_{i}\right) d_{i j}^{\alpha}\right) x_{j}+\beta_{i}-L^{-1}\left(\delta_{i}\right) \beta_{i}^{\alpha}\right]-\sum_{j=1}^{n}\left(c_{i j}+R^{-1}\left(\delta_{i}\right) c_{i j}^{\beta}\right) x_{j} \\
&+\Phi^{-1}\left(\gamma_{i}\right) \sqrt{\lambda_{i}^{2} \sum_{j=1}^{n} \sigma_{d i j}^{2} x_{j}^{2}+\lambda_{i}^{2} \sigma_{\beta i}^{2}+\sum_{j=1}^{n} \sigma_{c i j}^{2} x_{j}^{2}+\sigma_{\alpha i}^{2}}-\alpha_{i}-R^{-1}\left(\delta_{i}\right) \alpha_{i}^{\beta} \leq 0 .
\end{aligned}
$$

\section{Redundancy detection algorithm}

In this section, we build on the algorithms proposed by Charles et al. (2006, 2010), we present an algorithm to identify redundant fractional objective functions in multi-objective linear FS fractional programming problems. First, we need to recall the following definitions (Charles and Dutta, 2006; Charles et al., 2010).

\section{Definition 10:}

Let $\lambda$ be the following scalar:

$\lambda=\min \left\{\lambda_{i} \leq \frac{\sum_{j=1}^{n} \widetilde{\bar{c}}_{i j} x_{j}+\widetilde{\bar{\alpha}}_{i}}{\sum_{j=1}^{n} \widetilde{\bar{d}}_{i j} x_{j}+\widetilde{\bar{\beta}}_{i}} \mid i=1,2, \ldots, m\right.$ and $\left(x_{1}, x_{2}, \ldots, x_{n}\right)$ is the unit vector of $\left.\mathfrak{R}^{n}\right\}$.

The decision space relative to all the prob-pos constrained objective functions is defined by the following set:

$$
S^{O}=\left\{\begin{array}{l}
\mathbf{x} \in \mathfrak{R}^{n} \mid \lambda\left[\sum_{j=1}^{n}\left(d_{i j}-L^{-1}\left(\delta_{i}\right) d_{i j}^{\alpha}\right) x_{j}\right]-\sum_{j=1}^{n}\left(c_{i j}+R^{-1}\left(\delta_{i}\right) c_{i j}^{\beta}\right) x_{j}+\Phi^{-1}\left(\gamma_{i}\right) \sqrt{\lambda^{2} \sum_{j=1}^{n} \sigma_{d i j}^{2} x_{j}^{2}+\lambda^{2} \sigma_{\beta i}^{2}+\sum_{j=1}^{n} \sigma_{c i j}^{2} x_{j}^{2}+\sigma_{\alpha i}^{2}} \\
\leq \alpha_{i}+R^{-1}\left(\delta_{i}\right) \alpha_{i}^{\beta}-\lambda\left(\beta_{i}-L^{-1}\left(\delta_{i}\right) \beta_{i}^{\alpha}\right), i=1,2, \ldots, m, x_{j} \geq 0 \text { for } j=1,2, \ldots, n .
\end{array}\right\}
$$

For $w=1,2, \ldots, m$, the decision space relative to all the prob-pos constrained objective functions except the $\mathrm{w}$-th one is defined as follows:

$$
S_{w}=\left\{\begin{array}{c}
\mathbf{x} \in \mathfrak{R}^{n} \mid \lambda\left[\sum_{j=1}^{n}\left(d_{i j}-L^{-1}\left(\delta_{i}\right) d_{i j}^{\alpha}\right) x_{j}\right]-\sum_{j=1}^{n}\left(c_{i j}+R^{-1}\left(\delta_{i}\right) c_{i j}^{\beta}\right) x_{j}+\Phi^{-1}\left(\gamma_{i}\right) \sqrt{\lambda^{2} \sum_{j=1}^{n} \sigma_{d i j}^{2} x_{j}^{2}+\lambda^{2} \sigma_{\beta i}^{2}+\sum_{j=1}^{n} \sigma_{c i j}^{2} x_{j}^{2}+\sigma_{\alpha i}^{2}} \\
\leq \alpha_{i}+R^{-1}\left(\delta_{i}\right) \alpha_{i}^{\beta}-\lambda\left(\beta_{i}-L^{-1}\left(\delta_{i}\right) \beta_{i}^{\alpha}\right), i=1,2, \ldots, m, i \neq w, x_{j} \geq 0 \text { for } j=1,2, \ldots, n .
\end{array}\right\}
$$


Definition 11: For every $w=1,2, \ldots, m$, the constrained form of the $w$ th objective function is given by:

$$
\begin{array}{r}
\lambda\left[\sum_{j=1}^{n}\left(d_{w j}-L^{-1}\left(\delta_{w}\right) d_{w j}^{\alpha}\right) x_{j}\right]-\sum_{j=1}^{n}\left(c_{w j}+R^{-1}\left(\delta_{w}\right) c_{w j}^{\beta}\right) x_{j}+\Phi^{-1}\left(\gamma_{w}\right) \sqrt{\lambda^{2} \sum_{j=1}^{n} \sigma_{d w j}^{2} x_{j}^{2}+\lambda^{2} \sigma_{\beta w}^{2}+\sum_{j=1}^{n} \sigma_{c w j}^{2} x_{j}^{2}+\sigma_{\alpha w}^{2}} \leq \\
\alpha_{w}+R^{-1}\left(\delta_{w}\right) \alpha_{w}^{\beta}-\lambda\left(\beta_{w}-L^{-1}\left(\delta_{w}\right) \beta_{w}^{\alpha}\right)
\end{array}
$$

This constrained form is called redundant in model (3) if $S^{o}=S_{w}$.

It follows from Definition 11 that the constrained form of the wth objective function is redundant if and only if, for all $\mathbf{x} \in S_{w}$, we have:

$$
\begin{aligned}
& \lambda\left[\sum_{j=1}^{n}\left(d_{w j}-L^{-1}\left(\delta_{w}\right) d_{w j}^{\alpha}\right) x_{j}\right]-\sum_{j=1}^{n}\left(c_{w j}+R^{-1}\left(\delta_{w}\right) c_{w j}^{\beta}\right) x_{j}+ \\
& \Phi^{-1}\left(\gamma_{w}\right) \sqrt{\lambda^{2} \sum_{j=1}^{n} \sigma_{d w j}^{2} x_{j}^{2}+\lambda^{2} \sigma_{\beta w}^{2}+\sum_{j=1}^{n} \sigma_{c w j}^{2} X_{j}^{2}+\sigma_{\alpha w}^{2}} \leq \alpha_{w}+R^{-1}\left(\delta_{w}\right) \alpha_{w}^{\beta}-\lambda\left(\beta_{w}-L^{-1}\left(\delta_{w}\right) \beta_{w}^{\alpha}\right)
\end{aligned}
$$

Henceforth, we let:

$$
\begin{aligned}
& S_{w}(\mathbf{x})=\alpha_{w}+ R^{-1}\left(\delta_{w}\right) \alpha_{w}^{\beta}-\lambda\left(\beta_{w}-L^{-1}\left(\delta_{w}\right) \beta_{w}^{\alpha}\right)-\lambda\left[\sum_{j=1}^{n}\left(d_{w j}-L^{-1}\left(\delta_{w}\right) d_{w j}^{\alpha}\right) x_{j}\right]+ \\
& \sum_{j=1}^{n}\left(c_{w j}+R^{-1}\left(\delta_{w}\right) c_{w j}^{\beta}\right) x_{j}-\Phi^{-1}\left(\gamma_{w}\right) \sqrt{\lambda^{2} \sum_{j=1}^{n} \sigma_{d w j}^{2} X_{j}^{2}+\lambda^{2} \sigma_{\beta w}^{2}+\sum_{j=1}^{n} \sigma_{c w j}^{2} X_{j}^{2}+\sigma_{\alpha w}^{2}}
\end{aligned}
$$

and

$$
\bar{S}_{w}=\min \left\{S_{w}(\mathbf{x}) \mid \mathbf{x} \in S_{w}\right\}
$$

Definition 12: The constrained form of the wth objective function (8) is redundant in model (3) if $\bar{s}_{w} \geq 0$.

Definition 13: The constrained form of the wth objective function (8) is strongly redundant in model (3) if $\bar{s}_{w}>0$. Note that the constraint can be redundant without being strongly redundant. Definition 14: The constrained form of the wth objective function (8) is weakly redundant in model (3) if $\bar{s}_{w}=0$.

Using sequential LP, we start by linearizing the constrained version of the fractional objective function as defined in Section 4. In order to do so, we consider the constrained form of the $i$ th fractional objective function (see Definition 11), with $i=1,2, \ldots, m$, rewritten as follows: 


$$
\begin{aligned}
& f_{i}(\mathbf{x})=\lambda\left[\sum_{j=1}^{n}\left(d_{i j}-L^{-1}\left(\delta_{i}\right) d_{i j}^{\alpha}\right) x_{j}\right]-\sum_{j=1}^{n}\left(c_{i j}+R^{-1}\left(\delta_{i}\right) c_{i j}^{\beta}\right) x_{j}+ \\
& +\Phi^{-1}\left(\gamma_{i}\right) \sqrt{\lambda^{2} \sum_{j=1}^{n} \sigma_{d i j}^{2} x_{j}^{2}+\lambda^{2} \sigma_{\beta i}^{2}+\sum_{j=1}^{n} \sigma_{c i j}^{2} x_{j}^{2}+\sigma_{\alpha i}^{2}}-\left(\alpha_{i}+R^{-1}\left(\delta_{i}\right) \alpha_{i}^{\beta}\right)+\lambda\left(\beta_{i}-L^{-1}\left(\delta_{i}\right) \beta_{i}^{\alpha}\right) \leq 0
\end{aligned}
$$

Using Taylor's Formula for a function of $n$ variables, we have:

$\bar{f}_{i}(\mathbf{x})=f_{i}\left(\mathbf{x}^{*}\right)+\nabla f_{i}\left(\mathbf{x}^{*}\right) \cdot\left(\mathbf{x}-\mathbf{x}^{*}\right)^{T} \leq 0$

where $\nabla f_{i}\left(\mathbf{x}^{*}\right)$ is the gradient row vector of $f_{i}(\mathbf{x})$ at $\mathbf{x}^{*}=(1,1, \ldots, 1)$.

Since,

$$
\begin{aligned}
& f_{i}\left(\mathbf{x}^{*}\right)=\lambda\left[\sum_{j=1}^{n}\left(d_{i j}-L^{-1}\left(\delta_{i}\right) d_{i j}^{\alpha}\right)\right]-\sum_{j=1}^{n}\left(c_{i j}+R^{-1}\left(\delta_{i}\right) c_{i j}^{\beta}\right)+ \\
& +\Phi^{-1}\left(\gamma_{i}\right) \sqrt{\lambda^{2} \sum_{j=1}^{n} \sigma_{d i j}^{2}+\lambda^{2} \sigma_{\beta i}^{2}+\sum_{j=1}^{n} \sigma_{c i j}^{2}+\sigma_{\alpha i}^{2}}-\left(\alpha_{i}+R^{-1}\left(\delta_{i}\right) \alpha_{i}^{\beta}\right)+\lambda\left(\beta_{i}-L^{-1}\left(\delta_{i}\right) \beta_{i}^{\alpha}\right)
\end{aligned}
$$

and, for every $j=1,2, \ldots, n$, the $j$ th coordinate of the gradient vector $\nabla f_{i}\left(\mathbf{x}^{*}\right)$ is given by

$$
\frac{\partial f_{i}}{\partial x_{j}}\left(\mathbf{x}^{*}\right)=\lambda\left(d_{i j}-L^{-1}\left(\delta_{i}\right) d_{i j}^{\alpha}\right)-\left(c_{i j}+R^{-1}\left(\delta_{i}\right) c_{i j}^{\beta}\right)+\Phi^{-1}\left(\gamma_{i}\right) \frac{\lambda^{2} \sigma_{d i j}^{2}+\sigma_{c i j}^{2}}{\sqrt{\lambda^{2} \sum_{j=1}^{n} \sigma_{d i j}^{2}+\lambda^{2} \sigma_{\beta i}^{2}+\sum_{j=1}^{n} \sigma_{c i j}^{2}+\sigma_{\alpha i}^{2}}}
$$

we have:

$$
\begin{aligned}
& \nabla f_{i}\left(\mathbf{x}^{*}\right) \cdot\left(\mathbf{x}-\mathbf{x}^{*}\right)^{T}= \\
& =\left(\frac{\partial f_{i}}{\partial x_{1}}\left(\mathbf{x}^{*}\right), \frac{\partial f_{i}}{\partial x_{2}}\left(\mathbf{x}^{*}\right), \ldots, \frac{\partial f_{i}}{\partial x_{n}}\left(\mathbf{x}^{*}\right)\right) \cdot\left(x_{1}-1, x_{2}-1, \ldots, x_{n}-1\right)^{T}= \\
& =\lambda\left[\sum_{j=1}^{n}\left(d_{i j}-L^{-1}\left(\delta_{i}\right) d_{i j}^{\alpha}\right) x_{j}\right]-\sum_{j=1}^{n}\left(c_{i j}+R^{-1}\left(\delta_{i}\right) c_{i j}^{\beta}\right) x_{j}+\Phi^{-1}\left(\gamma_{i}\right) \frac{\sum_{j=1}^{n} \lambda^{2} \sigma_{d i j}^{2} x_{j}+\sum_{j=1}^{n} \sigma_{c i j}^{2} x_{j}}{\sqrt{\lambda^{2} \sum_{j=1}^{n} \sigma_{d i j}^{2}+\lambda^{2} \sigma_{\beta i}^{2}+\sum_{j=1}^{n} \sigma_{c i j}^{2}+\sigma_{\alpha i}^{2}}} \\
& -\lambda\left[\sum_{j=1}^{n}\left(d_{i j}-L^{-1}\left(\delta_{i}\right) d_{i j}^{\alpha}\right)\right]+\sum_{j=1}^{n}\left(c_{i j}+R^{-1}\left(\delta_{i}\right) c_{i j}^{\beta}\right)-\Phi^{-1}\left(\gamma_{i}\right) \frac{\sum_{j=1}^{n} \lambda^{2} \sigma_{d i j}^{2}+\sum_{j=1}^{n} \sigma_{c i j}^{2}}{\sqrt{\lambda^{2} \sum_{j=1}^{n} \sigma_{d i j}^{2}+\lambda^{2} \sigma_{\beta i}^{2}+\sum_{j=1}^{n} \sigma_{c i j}^{2}+\sigma_{\alpha i}^{2}}}
\end{aligned}
$$

and hence,

$$
\bar{f}_{i}(\mathbf{x})=f_{i}\left(\mathbf{x}^{*}\right)+\nabla f_{i}\left(\mathbf{x}^{*}\right) \cdot\left(\mathbf{x}-\mathbf{x}^{*}\right)^{T}=
$$




$$
\begin{gathered}
=\lambda\left[\sum_{j=1}^{n}\left(d_{i j}-L^{-1}\left(\delta_{i}\right) d_{i j}^{\alpha}\right) x_{j}\right]-\sum_{j=1}^{n}\left(c_{i j}+R^{-1}\left(\delta_{i}\right) c_{i j}^{\beta}\right) x_{j}+\Phi^{-1}\left(\gamma_{i}\right) \frac{\lambda^{2} \sigma_{\beta i}^{2}+\sigma_{\alpha i}^{2}}{\sqrt{\lambda^{2} \sum_{j=1}^{n} \sigma_{d i j}^{2}+\lambda^{2} \sigma_{\beta i}^{2}+\sum_{j=1}^{n} \sigma_{c i j}^{2}+\sigma_{\alpha i}^{2}}} \\
+\Phi^{-1}\left(\gamma_{i}\right) \frac{\sum_{j=1}^{n} \lambda^{2} \sigma_{d i j}^{2} x_{j}+\sum_{j=1}^{n} \sigma_{c i j}^{2} x_{j}}{\sqrt{\lambda^{2} \sum_{j=1}^{n} \sigma_{d i j}^{2}+\lambda^{2} \sigma_{\beta i}^{2}+\sum_{j=1}^{n} \sigma_{c i j}^{2}+\sigma_{\alpha i}^{2}}}-\left(\alpha_{i}+R^{-1}\left(\delta_{i}\right) \alpha_{i}^{\beta}\right)+\lambda\left(\beta_{i}-L^{-1}\left(\delta_{i}\right) \beta_{i}^{\alpha}\right) \leq 0
\end{gathered}
$$

We can rewrite inequality (9), for every $i=1,2, \ldots, m$, as follows:

$$
\bar{f}_{i}(\mathbf{x}) \leq\left(\alpha_{i}+R^{-1}\left(\delta_{i}\right) \alpha_{i}^{\beta}\right)-\lambda\left(\beta_{i}-L^{-1}\left(\delta_{i}\right) \beta_{i}^{\alpha}\right),
$$

where $\mathbf{x}=\left(x_{1}, x_{2}, \ldots, x_{n}\right)$, with $x_{j} \geq 0$, for every $j=1,2, \ldots, n$.

When $i$ ranges from 1 to $m$, inequality (9) gives place to a system of inequalities that can be interpreted in matrix form as follows:

$$
\bar{F} \cdot \mathbf{x} \leq \boldsymbol{\alpha}-\lambda \boldsymbol{\beta}
$$

where $\bar{F} \in \Re^{m \times n}$ and $\boldsymbol{\alpha}, \boldsymbol{\beta} \in \mathfrak{R}^{m}$ are defined by:

$$
\begin{aligned}
& \boldsymbol{\alpha}=\left(\alpha_{1}+R^{-1}\left(\delta_{1}\right) \alpha_{1}^{\beta}, \ldots, \alpha_{m}+R^{-1}\left(\delta_{m}\right) \alpha_{m}^{\beta}\right) \\
& \boldsymbol{\beta}=\left(\beta_{1}-L^{-1}\left(\delta_{1}\right) \beta_{1}^{\alpha}, \ldots, \beta_{m}-L^{-1}\left(\delta_{m}\right) \beta_{m}^{\alpha}\right) .
\end{aligned}
$$

By adding slack variables to the constraints form of the $m$ objective functions, premultiplying by the inverse of an appropriate basis and redefining the variables (both slacks and structural variables) as $x_{j}^{N B}$ (or) $x_{j}^{B}$ according to their status ( $N B$ for non-basic, and $B$ for basic), we obtain the following equivalence system:

$$
\left[\left(\bar{F}_{N B}\right)^{-1} \quad I\right]\left[\begin{array}{c}
\mathbf{x}^{N B} \\
\mathbf{x}^{B}
\end{array}\right]=\boldsymbol{\eta}
$$

where $\mathbf{x}^{N B}=\left(x_{1}^{N B}, \ldots, x_{k_{N B}}^{N B}\right)$ and $\mathbf{x}^{B}=\left(x_{1}^{B}, \ldots, x_{k_{B}}^{B}\right)$ are such that $x_{j}^{N B} \geq 0$ for every $j=1,2, \ldots, k_{N B}$ and $x_{j}^{B} \geq 0$ for every $j=1,2, \ldots, k_{B}$.

The matrix $\left(\bar{F}_{N B}\right)^{-1}$ is usually referred to as the Contracted Simplex Tableau (Dantzig, 1963). Let $\varphi_{i j}$ denote the $i j$ th element of the matrix $\left(\bar{F}_{N B}\right)^{-1}$. Also, let $\boldsymbol{\eta}$ be the "updated right hand side”, that is, $\left(\bar{F}_{N B}\right)^{-1}(\boldsymbol{\alpha}-\lambda \boldsymbol{\beta})$. 
Theorem 2 (Charles et al., 2006; 2010): A constrained form of the wth objective function is redundant if and only if its associated slack variable $s_{w}$ has the property $s_{w}=x_{t}^{B}$ in a basic solution where $\varphi_{t j} \leq 0$, for every $j=1,2, \ldots, n$, and $\eta_{t} \geq 0$.

Proof: If: In a basic solution, $x_{t}^{B}=\eta_{t}-\sum_{j=1}^{n} \varphi_{i j} x_{j}^{B}$. Since in any feasible solution the value of $x_{j}^{N B}$ will be at least zero, the sum is at least zero and, hence, $s_{w}=x_{t}^{B} \geq \eta_{t} \geq 0$. Therefore, $\overline{s_{w}} \geq 0$.

Only If: Let us consider the th row of the tableau as the objective function for the sequential LP $\bar{s}_{w}=\min \left\{S_{w}(\mathbf{x}) \mid \mathbf{x} \in S_{w}\right\}$. If $\overline{s_{w}} \geq 0$, it follows that in the optimal solution $\varphi_{t j} \leq 0$ for all $j=1,2, \ldots, n$ with $\eta_{t} \geq 0$. Since this optimal solution is a feasible extreme point of $S_{w}$, it is a basic feasible solution for the original set of constrained forms of the objective functions.

Note that, since in the theorem above $\bar{s}_{w}=\eta_{t}$, the constrained form of the wth objective function is strongly redundant if $\eta_{t}>0$ and weakly redundant if $\eta_{t}=0$.

The proposed redundancy detection algorithm is composed of seven steps as depicted in Figure 3:

\section{Insert Figure 3 Here}

1. Convert the FS fractional objective functions into constraints.

2. By using sequential LP (SLP), linearize the constrained form of the objective functions.

3. A matrix of intercept is constructed with decision and slack variables as rows and columns, respectively. This matrix is of order $m \times n$.

If $\eta_{t} \geq 0$, then

$\theta_{j i}=\frac{\left(\alpha_{i}+R^{-1}\left(\delta_{i}\right) \alpha_{i}^{\beta}\right)-\lambda\left(\beta_{i}-L^{-1}\left(\delta_{i}\right) \beta_{i}^{\alpha}\right)}{\bar{F}_{i j}} ; \bar{F}_{i j} \geq 0 ; i=1,2, \ldots, m ; j=1,2, \ldots, n$

$\bar{F}_{i j}$ denotes the $i j$ th element of the matrix $\bar{F}$

Else

$\theta_{j i}=\frac{\left(\alpha_{i}+R^{-1}\left(\delta_{i}\right) \alpha_{i}^{\beta}\right)-\lambda\left(\beta_{i}-L^{-1}\left(\delta_{i}\right) \beta_{i}^{\alpha}\right)}{\bar{F}_{i j}} ; \bar{F}_{i j}<0 ; i=1,2, \ldots, m ; j=1,2, \ldots, n$

$\bar{F}_{i j}$ denotes the $i j$ th element of the matrix $\bar{F}$ 
4. Identify the pivot element in each row.

If $\alpha_{i}+R^{-1}\left(\delta_{i}\right) \alpha_{i}^{\beta} \leq 0$, then

$\Psi_{j}=\max _{i}\left\{\theta_{j i}\right\}$,

Else

$\Psi_{j}=\min _{i}\left\{\theta_{j i}\right\}$, for all $j$ while the objective is maximum, vice versa.

5. Score out the row and column corresponding to the entering and leaving variables. If a column has more than one maximum/minimum, score out those rows also.

6. Remove these redundant constrained forms of fractional objective functions from the original model.

7. Solve the reduced multi-objective stochastic fractional programming problem to get the optimal solution with any mathematical programming solver.

\section{Numerical Examples}

In this section, two numerical examples (Examples 1 and 2) are presented to both demonstrate the applicability and exhibit the efficacy of the proposed redundancy algorithm for FS multi-objective fractional programming problems.

\subsection{Example 1}

$\operatorname{Max}\left[\frac{\widetilde{\bar{c}}_{11} x_{1}+\widetilde{\bar{c}}_{12} x_{2}+\widetilde{\bar{\alpha}}_{1}}{\widetilde{\bar{d}}_{11} x_{1}+\widetilde{\bar{d}}_{12} x_{2}+\widetilde{\bar{\beta}}_{1}}, \frac{\widetilde{\bar{c}}_{21} x_{1}+\widetilde{\bar{C}}_{22} x_{2}+\widetilde{\bar{\alpha}}_{21}}{\widetilde{\bar{d}}_{21} x_{1}+\widetilde{\widetilde{d}}_{22} x_{2}+\widetilde{\bar{\beta}}_{2}}\right]$

s.t.

$\operatorname{Pr}\left[\operatorname{Pos}\left[\tilde{\bar{a}}_{1} x_{1}+\tilde{\bar{a}}_{2} x_{2} \leq \tilde{\bar{b}}\right] \geq u\right] \geq p$

$5 x_{1}+2 x_{2} \leq 10$

$x_{1}, x_{2} \geq 0$

where $\alpha_{1}=\alpha_{2}=0, \beta_{1}=\beta_{2}=1$ and the confidence levels $u$ and $p$ are given by $u=0.5$ and $p=0.90$.

In Example 1, we consider two objectives, where all the coefficients are assumed to be FR numbers. Each of the FR coefficients has a symmetrical triangular membership function, which is a special case of a trapezoidal membership function. In fact, if in Eq. (1) we let $\alpha=\beta$, then the 
triangular fuzzy number is a symmetrical triangular fuzzy number. Hence, the membership functions for the coefficients in the example can be represented by pairs of the form $(\bar{m}, \alpha)$, where $m: N\left(u, \sigma_{m}^{2}\right)$ and $(\bar{m}, \alpha)$ is a symmetrical triangular fuzzy number. Note that $\bar{m}$ is the center and $\alpha$ is the spread. Table 1 provides the data used in Example 1.

\section{Insert Table 1 Here}

The deterministic equivalent of the constraint $\tilde{\bar{a}}_{1} x_{1}+\tilde{\bar{a}}_{2} X_{2} \leq \tilde{\bar{b}}$ is the following: $2 x_{1}+3 x_{2}+1.28 \sqrt{x_{1}^{2}+x_{2}^{2}+1} \leq 7$

The deterministic equivalent of the constrained form of the fractional objective functions is described below. This description is based on the confidence levels $\gamma_{1}, \gamma_{2}, \delta_{1}$ and $\delta_{2}$, that appear in the constrained form of the objective functions (i.e. the first set of constraints of model (3)), defined by:

$\gamma_{1}=0.10, \gamma_{2}=0.90$ and $\delta_{1}=\delta_{2}=0.5$.

$6 x_{1}+3 x_{2}-\lambda_{1}\left(5 x_{1}+2 x_{2}+1\right)+1.28 \sqrt{\left(2 \lambda_{1}^{2}+2\right) x_{1}^{2}+\left(\lambda_{1}^{2}+1\right) x_{2}^{2}} \geq 0$,
$16 x_{1}+11 x_{2}-\lambda_{2}\left(x_{1}+x_{2}+1\right)-1.28 \sqrt{\lambda_{2}^{2} x_{1}^{2}+\lambda_{2}^{2} x_{2}^{2}+x_{1}^{2}+x_{2}^{2}} \geq 0$.

Let $\lambda=\min \{1.125,9\}=1.125$ at $\left(x_{1}, x_{2}\right)=(1,1)$ from equations (11)-(12). Therefore, inequalities (11)-(12) reduce to (13)-(14):

$$
\begin{aligned}
& 0.375 x_{1}+0.750 x_{2}+1.28 \sqrt{4.531 x_{1}^{2}+2.266 x_{2}^{2}} \geq 1.125, \\
& 14.875 x_{1}+9.875 x_{2}-1.28 \sqrt{2.266 x_{1}^{2}+2.266 x_{2}^{2}} \geq 1.125 .
\end{aligned}
$$

Using the inequality (9) and SLP (Charles and Dutta, 2003, 2006; Charles et al., 2010), the following linear constraints are obtained:

$$
\begin{aligned}
& 2.6 x_{1}+1.863 x_{2} \geq 1.125, \\
& 13.513 x_{1}+8.513 x_{2} \geq 1.125
\end{aligned}
$$

\section{Insert Table 2 Here}

By using the proposed algorithm, it can be concluded from Table 2 that the second objective function is strongly redundant. Therefore, the problem can be solved by removing the second objective function from the original problem. The bi-objective FS fractional programming 
problem reduces to the following problem:

$\operatorname{Max} \quad 0.90 \lambda_{1}$

s.t.

$6 x_{1}+3 x_{2}-\lambda_{1}\left(5 x_{1}+2 x_{2}+1\right)+1.28 \sqrt{\left(2 \lambda_{1}^{2}+2\right) x_{1}^{2}+\left(\lambda_{1}^{2}+1\right) x_{2}^{2}} \geq 0$,

$2 x_{1}+3 x_{2}+1.28 \sqrt{x_{1}^{2}+x_{2}^{2}+1} \leq 7$,

$5 x_{1}+2 x_{2} \leq 10$,

$x_{1}, x_{2} \geq 0$.

The solution is obtained as follows: $x_{1}=1.547, x_{2}=0.000$ and $\lambda_{1}=2.384$.

\subsection{Example 2}

Let us consider the previous example along with a third objective function. That is:

$\operatorname{Max}\left[\frac{\widetilde{\bar{c}}_{11} x_{1}+\widetilde{\bar{c}}_{12} x_{2}+\tilde{\bar{\alpha}}_{1}}{\widetilde{\widetilde{d}}_{11} x_{1}+\widetilde{\bar{d}}_{12} x_{2}+\widetilde{\bar{\beta}}_{1}}, \frac{\widetilde{\bar{c}}_{21} x_{1}+\widetilde{\bar{c}}_{22} x_{2}+\tilde{\bar{\alpha}}_{21} \tilde{\bar{d}}_{1}+\widetilde{\bar{d}}_{22} x_{2}+\widetilde{\bar{\beta}}_{2}}{\frac{\widetilde{\bar{c}}_{31} x_{1}+\widetilde{\bar{c}}_{32} x_{2}+\widetilde{\bar{\alpha}}_{3}}{\widetilde{\bar{d}}_{31} x_{1}+\widetilde{\bar{d}}_{32} x_{2}+\widetilde{\bar{\beta}}_{3}}}\right]$

s.t.

$\operatorname{Pr}\left[\operatorname{Pos}\left[\tilde{\bar{a}}_{1} X_{1}+\tilde{\bar{a}}_{2} X_{2} \leq \tilde{\bar{b}}\right] \geq u\right] \geq p$

$5 x_{1}+2 x_{2} \leq 10$

$x_{1}, x_{2} \geq 0$

Let the confidence levels $\gamma_{1}, \gamma_{2}, \gamma_{3}, \delta_{1}, \delta_{2}$ and $\delta_{3}$, that appear in the constrained form of the objective functions (i.e. the first set of constraints of model (3)), be given by:

$$
\gamma_{1}=0.70, \gamma_{2}=0.90, \gamma_{2}=0.40 \text { and } \delta_{1}=\delta_{2}=\delta_{3}=0.5 \text {. }
$$

\section{Insert Table 3 Here}

The deterministic equivalent of the constrained form of the fractional objective functions is given below:

$$
\begin{aligned}
& 5 x_{1}+2 x_{2}-\lambda_{1}\left(5 x_{1}+2 x_{2}+1\right)-0.52 \sqrt{\left(2 \lambda_{1}^{2}+2\right) x_{1}^{2}+\left(\lambda_{1}^{2}+1\right) x_{2}^{2}} \geq 0, \\
& 16 x_{1}+11 x_{2}-\lambda_{2}\left(x_{1}+x_{2}+1\right)-1.28 \sqrt{\lambda_{2}^{2} x_{1}^{2}+\lambda_{2}^{2} x_{2}^{2}+x_{1}^{2}+x_{2}^{2}} \geq 0 .
\end{aligned}
$$


$11 x_{1}+13 x_{2}-\lambda_{3}\left(5 x_{1}+2 x_{2}+1\right)+0.25 \sqrt{2 \lambda_{3}^{2} x_{1}^{2}+\lambda_{3}^{2} x_{2}^{2}+2 x_{1}^{2}+x_{2}^{2}} \geq 4$

Let $\lambda=\min \{0.875,9,2.5\}=0.875$ at $\left(x_{1}, x_{2}\right)=(1,1)$ from equations (17)-(19). Therefore, inequalities (17)-(19) are converted to (20)-(22):

$0.625 x_{1}+0.25 x_{2}+1.28 \sqrt{3.531 x_{1}^{2}+1.766 x_{2}^{2}} \geq 0.875$,

$15.125 x_{1}+10.125 x_{2}-1.28 \sqrt{1.766 x_{1}^{2}+1.766 x_{2}^{2}} \geq 0.875$,

$6.625 x_{1}+11.25 x_{2}+0.25 \sqrt{3.531 x_{1}^{2}+1.766 x_{2}^{2}} \geq 4.875$.

By using SLP (Charles and Dutta, 2003, 2006; Charles et al., 2010), the following linear constraints are obtained:

$2.589 x_{1}+1.232 x_{2} \geq 0.875$

$13.299 x_{1}+8.424 x_{2} \geq 0.875$,

$7.009 x_{1}+11.442 x_{2} \geq 4.875$.

\section{Insert Table 4 Here}

By using the proposed algorithm, from the Table 4 it can be concluded that the second objective function is strongly redundant. Therefore, the problem is solved by removing the second objective function from the original problem. The tri-objective FS fractional programming problem can be converted into the following problem:

$\operatorname{Max} \quad 0.30 \lambda_{1}+0.60 \lambda_{3}$

$5 x_{1}+2 x_{2}-\lambda_{1}\left(5 x_{1}+2 x_{2}+1\right)+1.28 \sqrt{\left(2 \lambda_{1}^{2}+2\right) x_{1}^{2}+\left(\lambda_{1}^{2}+1\right) x_{2}^{2}} \geq 0$,

$16 x_{1}+11 x_{2}-\lambda_{2}\left(x_{1}+x_{2}+1\right)-1.28 \sqrt{\lambda_{2}^{2} x_{1}^{2}+\lambda_{2}^{2} x_{2}^{2}+x_{1}^{2}+x_{2}^{2}} \geq 0$,

$11 x_{1}+13 x_{2}-\lambda_{3}\left(5 x_{1}+2 x_{2}+1\right)+0.25 \sqrt{2 \lambda_{3}^{2} x_{1}^{2}+\lambda_{3}^{2} x_{2}^{2}+2 x_{1}^{2}+x_{2}^{2}} \geq 4$,

$2 x_{1}+3 x_{2}+1.28 \sqrt{x_{1}^{2}+x_{2}^{2}+1} \leq 7$

$5 x_{1}+2 x_{2} \leq 10$,

$x_{1}, x_{2} \geq 0$.

The solution is obtained as follows: $x_{1}=0.000, x_{2}=1.547, \lambda_{1}=1.503$ and $\lambda_{3}=4.444$. 


\section{Conclusion}

We considered the redundancy problem in a multi-objective linear FS fractional programming setting. Detecting and, consequently, eliminating redundant objective functions and/or constraints from the system under analysis is the key to avoid unnecessary calculations and reduce the computational complexity of the associated optimization algorithm.

Our analysis has focused on the fuzzy component characterizing many of the real-life situations where stochastic fractional programming can be applied and deliver significant results.

Despite the considerable literature on linear and nonlinear stochastic fractional programming, not much attention has been paid so far to FS fractional programming problems. The present paper shades some light in this direction developing an algorithm that allows to simultaneously identify redundant objective functions and redundant constraints in multi-objective linear FS fractional programming problems. More precisely, the proposed algorithm reduces the number of linear fuzzy fractional objective functions after transforming them in probabilisticpossibilistic constraints with respect to predetermined confidence levels.

The applicability of the proposed algorithm has been demonstrated by means of two numerical examples, where the existing redundancy has been removed and the number of linear fractional objective functions has been reduced accordingly. 


\section{References}

Ahmed, S., Elsholkami, M., Elkamel, A., Du, J., Ydstie, E.B., Douglas, P.L. (2014). Financial risk management for new technology integration in energy planning under uncertainty. Applied Energy, 128(1), 75-81.

Bajalinov, E.B. (2003). Linear-Fractional Programming: Theory, Methods, Applications and Software. Kluwer Academic Publishers, The Netherlands.

Birge, J.R. (1995). Models and model value in stochastic programming. Annals of Operations Research, 59, 1-18.

Birge, J.R., Louveaux, F. (2011). Introduction to Stochastic Programming. New York: Springer.

Charnes, A., Cooper, W.W. (1963), Deterministic equivalents for optimizing and satisfying under chance constraints. Operations Research, 11(1), 18-39.

Charnes, A., Cooper, W.W. (1962). Programming with linear fractional functionals. Naval Research Logistics Quarterly, 9 (3-4), 181-186.

Charles, V., Dutta, D. (2001). Linear stochastic fractional programming with branch-and-bound technique. In: Nadarajan, R., Kandasamy, P.R. (Eds.), National Conference on Mathematical and Computational Models. Allied Publishers, Chennai.

Charles, V., Dutta, D. (2003). Bi-weighted multi-objective stochastic fractional programming problem with mixed constraints. In: Nadarajan, R., Arulmozhi, G. (Eds.), Second National Conference on Mathematical and Computational Models. Allied Publishers, Chennai.

Charles, V., Dutta, D. (2006). Identification of redundant objective functions in multi-objective stochastic fractional programming problems. Asia-Pacific Journal of Operational Research, 23, 155-170.

Charles, V., Udhayakumar, A., Rhymend Uthariaraj, V. (2010). An approach to find redundant objective function(s) and redundant constraint(s) in multi-objective nonlinear stochastic fractional programming problems. European Journal of Operational Research, 201, 390398.

Dubois, D., Prade, H. (1980). Operations on Fuzzy Numbers. Fuzzy Sets and System: Theory and Applications. Academic Press, New York.

Dubois, D., Prade, H. (1988a). Fuzzy numbers: An overview. Analysis of Fuzzy Information, 2, 339.

Dubois, D., Prade, H. (1988b). Possibility Theory: An Approach to Computerized Processing of 
Uncertainty. New York: Plenum.

Dantzig, G. B. (1955). Linear programming under uncertainty. Management Science, 1, 197-206.

Dantzig, G.B. (1963). Linear Programming and Extensions. Princeton University Press, Princeton.

Dupacová, J. (2002). Applications of stochastic programming: Achievements and questions. European Journal of Operational Research, 140(2), 281-290.

Gal, T. (1975). A note on redundancy and linear parametric programming. Operational Research Quarterly, 26(4), 735-742.

Gal, T., Laberling, H. (1977). Redundant objective functions in linear vector maximum problems and their determination. European Journal of Operational Research, 1, 176-184.

Henrion, R. (2007). Structural properties of linear probabilistic constraints. Optimization, 56, 425440.

Henrion, R., Strugarek, C. (2008). Convexity of chance constraints with independent random variables. Computational Optimization and Applications, 41, 263-276.

Hirota, K. (1981). Concepts of probabilistic sets. Fuzzy Sets and Systems, 5, 31-46.

Jacob, V.C., Natesan, T.R., Rhymend Uthariaraj, V. (2002). Analysis of hybrid heuristic model reduction algorithms for solving linear programming problems. OPSEARCH, 39, 202-214.

Jeeva, M., Rajalakshmi, R., Charles, V., Yadavalli, V.S.S. (2004). An application of stochastic programming with Weibull distribution - cluster based optimum allocation of recruitments in manpower planning. Stochastic Analysis and Applications, 22(3), 801-812.

Kwakernaak, H. (1978). Fuzzy random variables. Part I: Definitions and theorems. Information Sciences, 15 (1), 1-29.

Kwakernaak, H. (1979). Fuzzy random variables. Part II: Algorithms and examples for the discrete case. Information Sciences, 17 (3), 253-278.

Liu, B. (1997). Dependent-chance programming: A class of stochastic programming. Computers \& Mathematics with Applications, 34(12), 89-104.

Liu, B. (1999). Uncertain Programming. New York: John Wiley \& Sons.

Liu, B. (2006). Introduction to Uncertain Programming. Physica-Verlag New York.

Liu, B. (2004). Uncertainty Theory: An Introduction to its Axiomatic Foundations, SpringerVerlag, Berlin.

Liu, B., Liu, Y.K. (2002). Expected value of fuzzy variable and fuzzy expected value models. IEEE Transactions on Fuzzy Systems, 10(4), 445-450. 
Pagnoncelli, B.K., Ahmed, S., Shapiro, A. (2009). Sample Average Approximation Method for Chance Constrained Programming: Theory and Applications. Journal of Optimization Theory and Applications, 142, 399-416.

Pal, B.B., Basu, I. (1995). A goal programming method for solving fractional programming problems via dynamic programming. Optimization, 35, 145-157.

Pal, B.B., Moitra, B.N., Maulik, U. (2003). A goal programming procedure for fuzzy multiobjective linear fractional programming problem. Fuzzy Sets and Systems, 139, 395405.

RhymendUthariaraj, V., Natesan, TR., Sankaranarayanan, V. (1999). Algorithms for identification of redundancies in linear programming problems. In The 19th IFIP Int. Conf. on Systems Modelling and Optimization, Cambridge, London.

Schaible, S. (1981). Fractional programming: applications and algorithms. European Journal of Operational Research, 7 (2), 111-120.

Wallace, S.W., Ziemba, W.T. (Eds.). (2005). Applications of Stochastic Programming, MPSSIAM Series on Optimization. SIAM.

Wets, R.J.B. (1996). Challenges in stochastic programming. Mathematical Programming, 75(2), 115-135.

Yang, N., Wen, F. (2005). Chance constrained programming approach to transmission system expansion planning. European Journal of Operational Research, 75(2-3), 171-177.

Zadeh, L.A. (1978). Fuzzy sets as a basis for a theory of possibility. Fuzzy Sets and Systems, 1(1), 3-28.

Zadeh, L.A. (1965). Fuzzy sets. Information and Control, 8 (3), 338-353.

Zarif, M., Javidi, M.H., Ghazizadeh, M.S. (2013). Self-Scheduling of large consumers with second-order stochastic dominance constraints. IEEE Transactions on Power Systems, 28(1), 289-299.

Zimmermann, H.J. (1996). Fuzzy set theory and its applications, 2nd Ed. Kluwer Academic Publishers, Dordrecht, The Netherlands. 


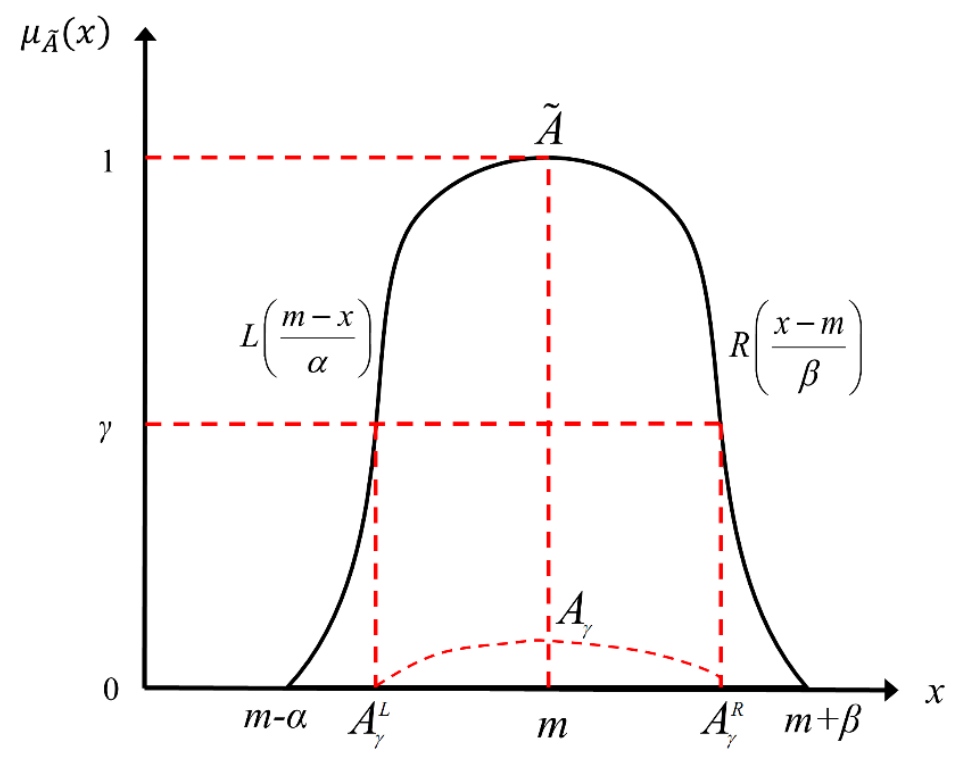

Figure 1. $\gamma$-cut of a generic L-R fuzzy number 


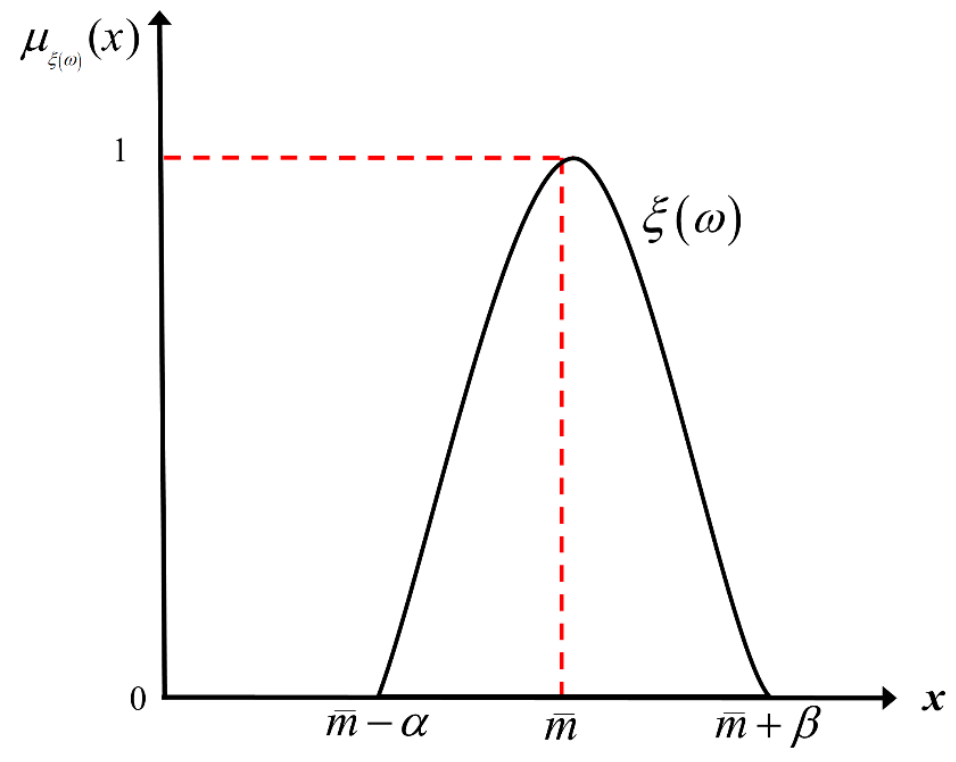

Figure 2. A generic L-R type FR variable 


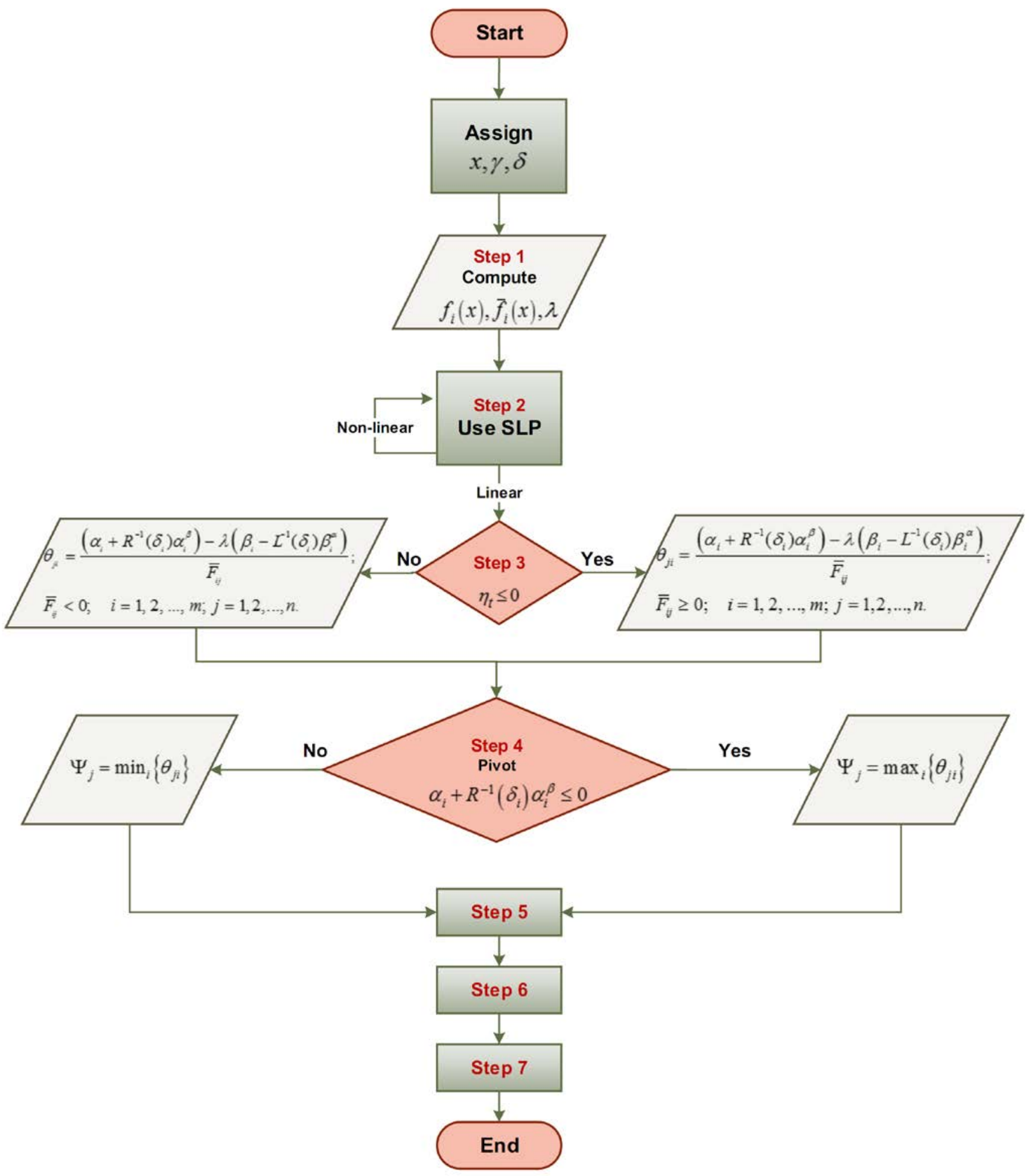

Figure 3. Redundancy detection algorithm 
Table 1. FR parameters and variables for Example 1

\begin{tabular}{|c|c|}
\hline Parameters & $\begin{array}{c}\text { Fuzzy } \\
\text { variables }\end{array}$ \\
\hline$\tilde{\bar{a}}_{1}$ & $(N(3,1), 2)$ \\
\hline$\tilde{\bar{a}}_{2}$ & $(N(5,1), 4)$ \\
\hline$\tilde{\bar{b}}$ & $(N(5,1), 4)$ \\
\hline$\tilde{\bar{C}}_{11}$ & $(N(5,2), 2)$ \\
\hline$\tilde{\bar{C}}_{12}$ & $(N(2.5,1), 1)$ \\
\hline$\tilde{\bar{C}}_{21}$ & $(N(15,1), 2)$ \\
\hline$\tilde{\bar{C}}_{22}$ & $(N(8,1), 4)$ \\
\hline$\tilde{\bar{d}}_{11}$ & $(N(6,2), 2)$ \\
\hline$\tilde{\bar{d}}_{12}$ & $(N(3,1), 2)$ \\
\hline$\tilde{\bar{d}}_{21}$ & $(N(1.5,1), 1)$ \\
\hline$\tilde{\bar{d}}_{22}$ & $(N(2,1), 2)$ \\
\hline
\end{tabular}


Table 2. Matrix-of-intercept for Example 1

\begin{tabular}{|c|c|c|c|}
\hline \multirow{2}{*}{$\begin{array}{c}\text { Decision } \\
\text { variables }\end{array}$} & \multicolumn{2}{|c|}{ Slacks } & Objective \\
\cline { 2 - 4 } & $\boldsymbol{s}_{\mathbf{1}}$ & $\boldsymbol{s}_{\mathbf{2}}$ & $\begin{array}{c}\text { function } \\
\text { value } \\
(\psi \boldsymbol{~})\end{array}$ \\
\hline $\boldsymbol{x}_{\mathbf{1}}$ & 0.433 & 0.083 & 0.433 \\
\hline $\boldsymbol{x}_{\mathbf{2}}$ & 0.604 & 0.132 & 0.604 \\
\hline
\end{tabular}


Table 3. FR parameters and variables for Example 2

\begin{tabular}{|c|c|}
\hline Parameters & $\begin{array}{c}\text { Fuzzy } \\
\text { variables }\end{array}$ \\
\hline$\tilde{\bar{c}}_{11}$ & $(N(4,2), 2)$ \\
\hline$\tilde{\bar{c}}_{12}$ & $(N(1.5,1), 1)$ \\
\hline$\tilde{\bar{c}}_{31}$ & $(N(10,2), 2)$ \\
\hline$\tilde{\bar{c}}_{32}$ & $(N(9,1), 6)$ \\
\hline$\tilde{\bar{d}}_{31}$ & $(N(6,2), 2)$ \\
\hline$\tilde{\bar{d}}_{32}$ & $(N(3,1), 2)$ \\
\hline$\tilde{\bar{\alpha}}_{3}$ & $(N(6,1), 4)$ \\
\hline$\tilde{\bar{\beta}}_{3}$ & $(N(2,1), 2)$ \\
\hline
\end{tabular}


Table 4. Matrix-of-intercept for Example 2

\begin{tabular}{|c|c|c|c|c|}
\hline \multirow{2}{*}{$\begin{array}{c}\text { Decision } \\
\text { variables }\end{array}$} & \multicolumn{3}{|c|}{ Slacks } & \multirow{2}{*}{$\begin{array}{c}\text { Objective } \\
\text { function } \\
\text { value } \\
(\psi)\end{array}$} \\
\hline & $s_{1}$ & $S_{2}$ & $S_{3}$ & \\
\hline$x_{1}$ & 0.338 & 0.066 & 0.696 & 0.696 \\
\hline$x_{2}$ & 0.710 & 0.104 & 0.426 & 0.710 \\
\hline
\end{tabular}

